a fragility fracture, a vast proportion of women at high risk remain untreated. Case-finding strategies prioritizing assessment of men and women with prior fracture are required to alleviate this problem.

Disclosure of Interest: None declared

DOI: 10.1136/annrheumdis-2017-eular.1859

\section{SP0118 ESTABLISHING AND IMPLEMENTING A FRACTURE LIAISON SERVICE}

S. Stephenson on behalf of National Osteoporosis Society. Service Development - Operations, National Osteoporosis Society, Bath, United Kingdom

Objectives: The objective of the National Osteoporosis Society (NOS) is to establish a Fracture Liaison Service (FLS) in every NHS Trust in the United Kingdom (UK). The Service Delivery Manager supports sites to establish, implement and develop a new FLS, as well as to improve the quality of existing services. The FLS model enables secondary fracture prevention through identification of fragility fractures in every person who breaks a bone aged over 50 using dedicated case-finding, with assessment and appropriate management of osteoporosis where necessary. The object of FLS is to prevent secondary fractures, in particular expensive hip and vertebral fractures, thereby providing both clinical and cost effectiveness for patients and payers. The NOS has developed a unique service to support FLS across the UK.

Developments: A team of specialist development managers with clinical and commissioning experience support providers and payers in the process of establishing new FLS's by offering consultation and guidance at every step of the process from pathway development to successful funding of services. This model has been replicated across the UK since April 2015 with the support and expertise of the NOS. Once an FLS is established the NOS provides support with service improvement, whether through additional commissioning of funds, Peer review or Gap analysis.

Results: Results from a range of analyses show that FLS has a positive impact on fracture rate and in particular hip fractures.

At the time of writing, the NOS is currently supporting 166 sites across the UK. 83 sites are improving the quality of their service; 58 sites are developing new services. 13 new services have been commissioned since commencement of the work programme, delivering new FLS provision to an additional 1.6 million people over 50 , preventing 1,482 hip fractures over a 5 -year period. Figures have been calculated from the NOS FLS Benefits Calculator https://benefits.nos.org.uk Challenges: The primary challenge in establishing an FLS is identifying a clinical champion - this maybe a nurse, an allied health professional, rheumatologist or ortho-geriatrician in the hospital, or a representative from Public Health or from a Clinical Commissioning Group (CCG). The champion can lead and take the FLS from an idea to implementation.

To support the establishment and implementation of FLS the NOS has developed the FLS Implementation Toolkit as well as the Clinical Standards for FLS. The Clinical Standards will shortly be supported with a supplementation - New Clinical Guidance on the identification of Vertebral Fractures.

Furthermore, the Charity has developed the Fracture Prevention Practitioner (FPP) training for those wishing to implement an FLS. This is backed by the Competency Framework for Nurses, allied health professional and doctors to ensure best practice in fracture prevention.

Conclusion: The NOS service development model of support is successful in driving the establishment, implementation and improvement of FLS across the UK. This is tough in an economic climate where health budgets are constrained. However, there is strong evidence that investment in FLS improves the quality of care as well as illustrating financial savings in health and social care. NHS England recommends that every patient with/or at risk of osteoporosis and fragility fractures should have access to a commissioned service.

Disclosure of Interest: None declared

DOI: 10.1136/annrheumdis-2017-eular.7217

\section{SP0119 PREDICTING FRACTURE RISK: ACCURACY AND FEASIBILITY OF TOOLS}

A.A. Marques. Rheumatology, Centro Hospitalar e Universitario de Coimbra, Coimbra, Portugal

Several therapeutic options and screening strategies are available to effectively decrease fracture risk. However the main clinical challenge still consists in accurately identifying and selecting individuals for bone densitometry and for pharmacological treatment, in order to increase efficiency and minimize individual and societal costs.

The World Health Organization provided an operational definition of osteoporosis as a bone mineral density (BMD) that lies 2.5 or more standard deviations below the average value for young healthy women of the same gender and ethnical background [T-score $\leq-2.5]$. However, BMD has limited sensitivity and specificity in the prediction of fracture. In fact, a large number of conditions have been firmly established as risk factors for the occurrence of fragility fractures, independently from BMD. These have been combined into prediction algorithms to estimate fracture probability and are currently available for calculate the risk of fractures. However, the existing tools differ in many relevant aspects: from their own feasibility, to the number and availability of clinical risk factors included, the accessibility of BMD measurements and, finally, their performance in different settings.

With this session we aim to identify and synthesize the best available evidence on the accuracy and feasibility of the currently available tools designed to predict fracture risk.

Several therapeutic options and screening strategies are available to effectively decrease fracture risk. However the main clinical challenge still consists in accurately identifying and selecting individuals for bone densitometry and for pharmacological treatment, in order to increase efficiency and minimize individual and societal costs.The World Health Organization provided an operational definition of osteoporosis as a bone mineral density (BMD) that lies 2.5 or more standard deviations below the average value for young healthy women of the same gender and ethnical background [T-score $\leq-2.5]$. However, BMD has limited sensitivity and specificity in the prediction of fracture. In fact, a large number of conditions have been firmly established as risk factors for the occurrence of fragility fractures, independently from BMD. These have been combined into prediction algorithms to estimate fracture probability and are currently available for calculate the risk of fractures. However, theexisting tools differ in many relevant aspects: from their own feasibility, to the number and availability of clinical risk factors included, the accessibility of BMD measurements and, finally, their performance in different settings. With this session we aim to identify and synthesize the best available evidence on the accuracy and feasibility of the currently available tools designed to predict fracture risk.

Disclosure of Interest: None declared

DOI: 10.1136/annrheumdis-2017-eular.7255

\section{SP0120 PREDICTING THE RISK OF FALLS AND PROMOTING BALANCE IN OLDER ADULTS}

\section{Rose. Kinesiology, California State University, Fullerton, Fullerton, United} States

Falls are the leading cause of injury and death due to injury among the older adult population in the United States. Of those who fall, $24 \%$ will sustain serious injuries and $6 \%$ will experience fractures. In addition to injury, older adults who fall may experience decreased functional ability, loss of independence, a poorer quality of life, or premature mortality. At a global level, these statistics are similar among developed countries. It is important to first identify the intrinsic and extrinsic risk factors that contribute to falls and then intervene appropriately once the level of fall risk has been identified. It is important to understand that there is no one size suits all fall risk reduction program and that the type of program will vary as a function of the level of risk. Core components of successful fall risk reduction programs include exercise, environmental modifications, and behavior change techniques aimed at fostering long-term adherence to engaging in fall risk reducing behaviors. The purpose of this presentation will be to describe appropriate methods for identifying fall risk, and intervention strategies that have been shown to significantly reduce fall risk and/or fall incidence rates across the continuum of fall risk.

Disclosure of Interest: None declared

DOI: 10.1136/annrheumdis-2017-eular.7244

\section{FRIDAY, 16 JUNE 2017}

\section{Biological agents in juvenile idiopathic arthritis: open issues}

\section{SP0121 LONG TERM SIDE EFFECTS OF BIOLOGICAL AGENTS IN JIA}

J.F. Swart on behalf of Pharmachild and Foreum (Comorbidity in JIA). Pediatric Rheumatology, UMC Utrecht, Utrecht, Netherlands

In the last years, the interest in the concept of comorbidity and its societal as well as individual impact has increased. Juvenile idiopathic arthritis (JIA) is a chronic inflammatory disease starting in childhood which often persists into adulthood. Clinicians are facing an aging population with multiple morbid conditions occurring in one individual. Long term outcome studies show the high prevalence and the potential interaction of coexisting diseases. For JIA recent studies reported that uveitis, asthma/atopic diseases and diabetes mellitus are prevalent comorbidities in JIA with $11.6-30 \%, 10.8 \%$ and $3.5 \%$ respectively, followed by cardiovascular disease, malignancies and inflammatory bowel diseases. Childhood long term outcome studies and Pharmacovigilance registries already revealed associations of co-existing diseases and the role of used medication (especially biologicals). It is important to plan preventive and screening strategies in order to prevent or early detect and treat comorbidities and integrated follow up once comorbidity exists.

Disclosure of Interest: None declared

DOI: 10.1136/annrheumdis-2017-eular.7172 